\title{
SOME POINTS IN THE PREVENTION OF DIPHTHERIA INFECTION.*
}

\author{
By A. GRIFFITH, M.D., \\ Medical Officer of Health for Hove.
}

THE object of this paper is to raise a few practical points for discussion, and to learn what the members of the branch are doing in certain directions, that we may if possible get more light to enable us to grapple with this disease, about which we know as much, if not more, than of any other, and which nevertheless seems to maintain its vitality in spite of our persistent attacks. I say we know more of this disease than of any other infectious disease because we can and do actually handle-at the end of a platinum needle-the materies morbi to the destruetion of which all our efforts are directed.

It is such a comforting assurance to our patients and to general practitioners to know that diphtheria is due to the Klebs-Löffler bacillus; but, like all advances in knowledge, this one only leads us to further questions. The difficulty at once arises that there are several varieties of this bacillus, and we are puzzled to know the meaning of each. Well, we are getting nearer to that, and sometimes one is almost hopeful that the varieties are true clinically as well as in the laboratory.

The usual three varieties, which you all know, are the long, the short, and the pseudo, of which the first two seem better under. stood than the last; for example, you get a smart outbreak in a school with several consecutive cases, you may be pretty sure that the bacteria are of the long variety; or, in extreme contrast to this, a single case arises from no one knows where, and two or three mild sore throats follow in the house; the short bacillus will be present in the late throats.

As to the pseudo-diphtheria bacillus one can only say that the opinions of different observers seem to be very divergent.

But even of these well-defined groups there are modifications. I dare say those of you who do bacteriology have been struck at times by a very large, fat, and well-staining-in-parts bacillus, which suggests a caterpillar after a good meal, and which seems to be of peculiarly unpleasant ferocity. I have noticed it in one instance where the disease ran quickly through a family of four 1902.

*Paper read at the meeting of the Southern Branch of the Society on July 3rd, 
persons, and in two other very severe attacks, which both ended fatally, the second of which was caught from the first with an incubation period clearly defined as ten days. In each of these it. occurred in a pure culture, and showed a very rapid growth on blood serum. Since I wrote this, I have discovered in conversation that in some of the London laboratories there are treasured cultures of a very large bacillus, from which the earlier injections into horses were made, which was of exceptional virulence, thus forming an interesting confirmation of my own local experience. With regard to the short bacilli, one fact, probably well known, is worth mention. I have found sometimes, after incubating a bloodserum specimen of a swab for sixteen hours, oval bacilli suggestive of diphtheria, which can only be returned as doubtful or suspicious ; by the next day they are ordinary short diphtheria bacilli. So frequently has this happened that when I get them now I say that I will report definitely on the morrow. Then there are the forms we get in convalescents, viz., the polar, and one very like the pseudo, which in fact we should call so if we had not watched the gradual alteration of them, swab by swab, from the original long form. Of course it may be said that in such instances the pseudo-diphtheria. bacilli only appear when the true ones have died out; but circumstantial evidence is certainly in favour of the gradual degeneration.

We want to know the exact significance of each kind of bacillus, which at present we do not know, and until the varieties are clearly defined, much research work is contradictory. In cases, for example, where a large number of ill or healthy persons are swabbed and diphtheria bacilli are found, we have gained little information until we know of what kind they are, and whether virulent or not.

It appears that the virulent and non-virulent bacilli are indistinguishable except by actual inoculation into animals, a practice which it is impossible to apply in ordinary cases. We are much in want of some test for virulence, but until we get it we must go on the principle that any bacilli which grow quickly and stain well are dangerous.

Of course we cannot doubt this if they are obtained from an inflamed throat; it is when we get them in convalescent patients and in "contacts" that the difficulty and the importance of deciding at once arise.

How long are we to keep diphtheria patients in isolation hospitals? There seems to be a divided opinion on this. For my own part I act on the principle of keeping patients under control in hospital until all diphtheria bacilli have gone-i.e., until a throat-swab does not, when incubated, produce any. In some cases two negatives 
are required, but I should be only too glad to believe that I might with safety discharge them sooner.

I have three times only, knowingly, sent home patients with diphtheria bacilli of active growth in their throats, all being adults. In one instance fresh cases arose five weeks later in the house, the patient having had a "fresh cold" in the meantime.

In spite of the practice of some hospital medical officers who say it is absurd to keep patients so long, I cannot persuade myself that there is no danger of infection when I get from the throat the same result as I obtained in the acute stage of the disease. I believe that the return cases occurring after diphtheria and scarlet-fever are almost all explained by the persistence of infection in the throat, and that when we are able to recognise the scarlet-fever germ, we shall find that in these cases the microbes remain for long periods in the throat, as we know that they do in diphtheria.

Anything which will lessen or abolish these return cases must be welcomed, and to knowingly discharge patients who may be dangerous seems to me to be out of line with our usual action as public health officers. I may say that besides the adult mentioned as causing return eases, only one other patient has given the disease to others at home out of over 200 who have passed out of the hospital.

Another question I would ask is, What can be done to destroy more quickly the bacilli in the throat, or to render them harmless?

I have tried three methods, viz. :

1. Complete isolation and open-air treatment by day.

2. Antisepties of various kinds.

3. Repeated injections of anti-toxin.

There can be no doubt that the open air and complete separation from other diphtheria patients is most beneficial; yet it often appears to produce no effect. I have separated a patient for seven or eight weeks, and found the bacilli as active in growth and as abundant as ever.

My own impression is that patients not only recover more quickly, but lose their infectiousness sooner when they are nursed at home, and this because they are truly isolated. As a contrast to this I have a few times had relapses in convalescent patients, when others with fresh illness have been admitted to the same ward. One case was partieularly clear in its significance-when the patients already mentioned with the very large bacilli came into the ward, one girl who had been there for twelve days with a mild attack had a second bad throat with membrane, and the same large bacilli were obtained from her throat. 
The most rapid clearing of infection I have yet met with occurred in a woman recently under observation, who had a very slight inflammation of the throat, in which the long bacilli were found in abundance. She was going about out of doors freely all the time: in eight days the bacilli were polar, and on the twelfth were not to be found.

Antiseptics are regularly employed at our hospital throughout; in cases without adenoids or large tonsils the throats clear fairly quickly; but in many even a nasal douche at frequent intervals seems to produce no effect on the bacilli. I have tried silver nitrate in 4 per eent. solution, repeated daily to the whole pharynx; eucalyptus applications of almost full strength; keeping the patient in a room impregnated with eucalyptus; and lysol and perchloride of mercury ad libitum. I am now using 1 per cent. solution of carbonate of ammonium to clear the throat first, and a solution of chinosol afterwards.

The only thing I can say is that weak antiseptics do better than those which irritate; and that when their use is suspended the bacilli grow with greater activity than during their application.

In a few instances-only nine-I have tried a repeated injection of anti-toxin (Behring's) during the stage of convalescence, when I was in despair of getting rid of the bacilli. It did not produce any striking or immediate result.

Mention of anti-toxin as a bacilli killer raises another point as to its use as a prophylactic. What is its mode of action? The results are fairly satisfactory, and the records of large numbers prove that the percentage of secondary cases from infected houses is much less where anti-toxin has been used; its value is emphasised by the fact that it is generally given only in the worst cases in which infection is likely to spread.

Now it can only act in two ways, either by destroying the bacilli, or by supplying the recipients with an antidote to the toxins which they produce.

It has been stated that it renders the throat a less congenial habitat for bacilli, and that it prevents their multiplication, if it does not destroy them.

But experience shows that it hinders their growth very little, if at all. The experience of Matson, of Pittsburg, recorded in Public HEALTH, ${ }^{*}$ is that, though a large dose prevents the development of the disease in "contacts," the bacilli are often long in disappearingas long as from persons who have actually had diphtheria. 
In order to test the direct effect of anti-toxin upon vigorous diphtheria bacilli, I recently made a few mixtures of sterile broth and Behring's serum in varying proportions, and into each I put some long diphtheria bacilli. The specimens were incubated for two days, and loops were taken from each and drawn over sterile blood serum. The results, to put them briefly, were that in a mixture of equal parts of anti-toxin and broth, bacilli were found of indefinite outline and taking a poor stain, which grew on the blood serum, but retained their feeble characteristics. _ After standing for a week, they would not grow on blood serum.

In the mixture of 1 to 8 they seemed to multiply freely, and at the end of a week grew well on agar agar. It is possible that they had lost their virulence, but this I was unable to test.

It seems possible, then, that diphtheria bacilli will grow in a mixture of anti-toxin diluted eight times; and this being so, it is difficult to think that the small amount of it we inject can affect the bacilli directly.

There is a practical issue to this, viz.: If this prophylactic serum only renders the recipient immune, and that for a short time, and does not affect the bacilli themselves, are not such persons a danger to others? Is it enough to give the serum, prevent the appearance of clinical diphtheria, and allow the persons to get about among others?

The only way we can get exact views is to test whether there are bacilli in the throat when the prophylactic dose is given, and to see how long they persist. We shall then be better able to judge whether it destroys them or not. This method has been carried out in schools on several occasions: all those with bacilli in their throats were separated from others, and were injected; as the bacilli disappeared from one and another they were discharged from supervision. Someone has recently advocated repeating the anti-toxin every two or three weeks while bacilli continue; and this seems reasonable, for is it not possible that in cases who have developed diphtheria three or four weeks after injection, the bacilli have been all the time in the throat, and have only waked to activity when the antidote has ceased to act? The truth seems to be that while prophylactic doses of anti-toxin are exceedingly useful in preventing the development of diphtheria in persons exposed to infection, they do not diminish the risk to others associating with them. The only way to prevent the disease spreading is to keep away from others all those who have been exposed to danger and have bacilli in their throats, whether clinically ill or not. 
The ideal method is to swab all contacts and to see that they are free of bacilli, or to isolate them until they are. What degree of isolation will be necessary depends much on circumstances; at least they should remain away from school and close association with others indoors.

While this may be rather impracticable on account of the amount of work involved, we are doing the nearest we can to it by excluding eontacts from school for a certain time. My own rule until lately has been to exclude for two weeks after disinfection was done; but I have recently lengthened the period to four weeks, and so far with most satisfactory results.

One more point as to the ventilation of board schools and private schools. It has lately been much advocated that children under five years should be kept away from school, and I think we all agree that little would be lost, except ill health; but if they do go, the rooms in which they gather ought to be exceptionally well ventilated and heated.

Something should be done to create a higher standard for board schools, especially of the infant classes ; and I think it is a matter which our society might take up.

Combustrbla Gases of the Atmosphere-Atmosphertc Hronogen. A. Gavtier (Ann.Chim. et Phys., 7 sér., 22, January, 1901, pp. 5-110; Experiment Station Record, vol. xiii., No. 4) concludes from the long series of investigations of air freed from suspended matter, here reported, that there exists normally in pure air about 20 parts of free hydrogen to 100,000 parts of air, as well as \& certain amount of hydrocarbons due to exhalations from the soil, from plants, fermentations, manufactories, etc. They, however, appear to diminish as the air becomes purer. They are found in comparatively large quantities in the air of towns, to a less extent in that of the country, in very small amounts in the air of rocky plateaus and the peaks of high mountains, and are entirely absent from the pure air derived from the high regions of the atmosphere.

The character and proportions of the combustible gases found in the air are indicated by the following analysis of the air at Paris, which may be taken as representative of the air of large cities in general : 100 litres of the air of Paris at $0^{\circ}$ and 760 millimetre pressure contain free hydrogen, 19.4 c.c.; methane, 121 c.c.; benzin $\left(\mathrm{C}_{6} \mathrm{H}_{6}\right)$ or similar hydrocarbons, 1.7 c.c. ; carbon monoxide, with traces of various hydrocarbons, $0 \cdot 2$ c.c.

M. Gautier has also come to the conclusion (Bul. Soc. Chim. Paris, 3 sér., 25, 1901, No. 5, pp. 231, 232) that the hydrogen of the air is of volcanic origin, being produced by the action of igneous rocks on aqueous vapour. 